\title{
Prediction of fertility by centrifugal countercurrent distribution (CCCD) analysis: correlation between viability and heterogeneity of ram semen and field fertility
}

\author{
R. Pérez-Pé1, J. I. Martí1, E. Sevilla², M. Fernández-Sánchez³, \\ E. Fantova ${ }^{4}$, J. Altarriba ${ }^{5}$, J. A. Cebrián-Pérez ${ }^{1}$ \\ and T. Muiño-Blanco ${ }^{1 *}$ \\ Department of ${ }^{1}$ Biochemistry and Molecular and Cell Biology, and ${ }^{5}$ Department of Anatomy \\ and Genetics, School of Veterinary Medicine, University of Zaragoza, Miguel Servet 177, \\ 50013-Zaragoza, Spain; ${ }^{2}$ CENSYRA (DGA), ${ }^{3}$ ANGRA and ${ }^{4}$ Carnes-Oviaragón SCL, \\ Zaragoza, Spain
}

The prediction of the fertilizing ability of a sire or a given insemination dose is a primary aim in the field of artificial insemination. Centrifugal countercurrent distribution analysis (CCCD) was used to determine the relationship between some sperm parameters and the in vivo fertility rate obtained with the same sample after cervical artificial insemination. A total of 522 ewes from 26 different farms was inseminated with 53 ejaculates obtained from 25 mature Rasa aragonesa rams. Semen was diluted to $1.6 \times 10^{9}$ cells ml-1 and doses of $0.25 \mathrm{ml}$ were prepared and kept at $15^{\circ} \mathrm{C}$ until used for insemination. The same ejaculates were used for analysis of standard semen parameters and CCCD analysis. Sperm motility, concentration and viability were determined before and after CCCD. Post-CCCD parameters were derived from the analysis of the profile obtained after CCCD. The recovered viability showed the highest correlation with fertility, especially in the central chambers $\left(V_{2}, r=0.415, P<0.005\right)$. The ejaculate heterogeneity also showed a positive correlation with field fertility $(r=0.23)$, with a tendency towards significance $(P<0.1)$. The mean fertility value of all ejaculates used in this study was $46.75 \%$, ranging from $12.5 \%$ to $75.0 \%$. Ejaculates were classified into two categories according to their fertility: higher and lower than the mean value. Only the viability recovered in the central chambers $\left(V_{2}\right)$ was a parameter with a predictive capacity to discriminate between the two groups $(P<0.05)$. A predictive equation for field fertility with a correlation coefficient $r=0.488$ and a very high level of significance $(P<0.005)$ was deduced by multiple analysis: $P F=6.02+0.069 V_{2}+0.315 H$ (where $P F$ is predictive fertility, $V_{2}$ is the recovered viability in the CCCD profile central chambers and $H$ is heterogeneity).

\section{Introduction}

Before including a male in an artificial insemination programme, it is important to know its potential fertility. Although the fertility of a sire is often evaluated after insemination of a certain number of females, this is expensive and it takes time to perform a representative number of inseminations. Therefore, the availability of other methods for the accurate prediction of the fertilizing ability of a sire or a given insemination dose is a primary aim in the field of artificial insemination.

Over the years, numerous methods have been developed for this purpose. Several laboratory tests that analyse different aspects of semen quality have been correlated with field fertility. These assays can be classified as basic semen parameter analysis and sperm function assays. Standard semen

*Correspondence

Email: muino@posta.unizar.es analysis assays include assessment of sperm morphology (Rogers et al., 1983; Barth, 1992), sperm motility (Kjaestad et al., 1993; Holt et al., 1997), presence of intact acrosomes (Correa et al., 1997) and membrane integrity (Pérez et al., 1997). Significant correlations between motility and field fertility have been found by some workers (Kjaestad et al., 1993; Correa et al., 1997) but not by others (Graham et al., 1980; Söderquist et al., 1991; Januskauskas et al., 1996). An inverse relationship between the number of morphologically abnormal spermatozoa and fertility has been reported (Barth et al., 1992), although contradictory results about correlation between membrane integrity and fertility have been found (Eriksson et al., 1994; Januskauskas and Rodriguez-Martinez, 1995; Correa et al., 1997; Garner et al., 1997).

Several assays have been developed in the last two decades to evaluate sperm function. They include the sperm penetration assay (Tatemoto et al., 1994; Gadea et al., 1998), the zona binding (Ivanova and Mollova, 1993; Meyers, 1996; Gadea, et al., 1998; Zhang et al., 1998) or hemi-zona (Fazeli et al., 1995) assays, and several forms of acrosome reaction 
assay (Whitfield and Parkinson, 1995; Januskauskas et al., 1999). These tests provide information about the ability of spermatozoa to interact with the oocyte, and some significant correlation between them and in vivo fertility has been found in several species (pigs and cattle: Fazeli et al., 1995, 1997).

As fertilization is a process that requires several sperm functions, it seems logical that the combination of a multiple parameter assay, derived from the application of several evaluation tests, could achieve a more precise prediction of the fertilizing ability of a semen sample, as suggested by Amann and Hammerstedt (1993). These combinations could include only parameters derived from standard semen analysis or combined with others based on sperm function assays.

As many of the steps that occur during fertilization depend on changes in sperm surface, partition in aqueous two-phase systems can be very useful in the analysis of sperm surface characteristics related to fertility. This technique is based on different cell surface affinities for inmiscible aqueous solutions of polymers, such as dextran and polyethylene glycol (PEG) (Albertsson, 1986; Fisher and Sutherland, 1989). The upper phase is rich in PEG and is relatively more hydrophobic than the lower phase, which is rich in dextran. When cells are added to the system they partition between the interface and the PEG-rich upper phase. The extent of partition is dependent on the cell surface properties. Thus, in the same two-phase system, cells with different surface characteristics will partition to a different extent.

The selectivity and separation resolution can be improved several fold by using multi-step partition procedures. Countercurrent distribution (CCD) is a chromatographic process with one stationary (lower) phase and one mobile (upper) phase. The cell sample is partitioned in one system and the two phases are then, in a systematic way, brought into contact with fresh opposite phases. The loss of viability as a result of dilution (Harrison et al., 1982) and washing during the separation process can be avoided by including centrifugation (CCCD: centrifugal countercurrent distribution).

CCCD in an aqueous two-phase system is a resolutive technique that reveals sperm heterogeneity (García-López, 1996; Ollero et al., 1997), which is involved extensively in successful fertilization (Amann and Hammerstedt, 1993). In the present study, the relationship between some standard parameters of spermatozoa and other parameters derived from the analysis of the semen sample by CCCD were investigated, and the in vivo fertility rate obtained with the same sample after cervical artificial insemination was determined. The data have been examined statistically to determine the correlations between these CCCD parameters, both individually and combined with standard semen analysis parameters, and field fertility.

\section{Materials and Methods}

\section{Reagents}

Dextran T-500 (M 500 000) and Ficoll $400\left(M_{r} 400000\right)$ were obtained from Pharmacia (Uppsala). Polyethylene glycol (M 6000; PEG) was purchased from Serva Feinbiochemica (New York, NY). All other chemicals used were of analytical reagent grade.

\section{Sperm collection}

All the experiments were performed using fresh ram spermatozoa. Semen samples were obtained from 25 mature Rasa aragonesa rams maintained at the Centro de Selección y Reproducción Animal de la Diputación General de Aragón (CENSYRA, Zaragoza). Semen samples were collected using an artificial vagina, within the regular collecting schedule at the station. For each experiment, an aliquot of two different ejaculates was taken and kept at $30^{\circ} \mathrm{C}$ until evaluation, usually performed within $1 \mathrm{~h}$ after collection. Both samples were analysed simultaneously in each experiment in identical conditions.

The artificial inseminations were performed by the technical teams of the National Association of Rasa Aragonesa Breeding (ANGRA) and Carnes Oviaragón SCL on many different farms owned by these companies. Each

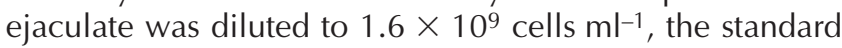
sperm concentration used in commercial artificial breeding centres with the Rasa aragonesa breed. The diluted samples were packaged in $0.25 \mathrm{ml}$ straws and kept at $15^{\circ} \mathrm{C}$ until used for insemination (between $3-5 \mathrm{~h}$ after collection, depending on the distance to the farm).

\section{Standard semen analysis}

Sperm concentration. Sperm concentration was calculated in duplicate using a Neubauer's chamber (Marienfeld, Lauda-Königshofen).

Sperm motility. Motility evaluations were performed by the same person throughout the study and were assessed subjectively by visual estimation under a phase-contrast microscope. Mass motility was assessed at $\times 10$ magnification by placing $10 \mu \mathrm{l}$ of undiluted semen on a slide glass maintained at $37^{\circ} \mathrm{C}$, scoring from 1 to 5 according to the wave movement activity. Semen samples obtaining a score of $<4$ were rejected. Progressive individual motility was evaluated at $\times 100$ magnification by placing $5 \mu$ of semen diluted 1 in 100 with the CCCD polymer-free medium on a glass slide under a coverslip, maintained at $37^{\circ} \mathrm{C}$.

Membrane integrity. Cell viability (membrane integrity) was assessed by fluorescent staining with carboxifluorescein diacetate and propidium iodide (Harrison and Vickers, 1990). The cells were examined under a Nikon fluorescence microscope, and the number of propidium iodide-negative (membrane-intact) and propidium iodidepositive (membrane-damaged) spermatozoa per 100 cells was estimated. The assay of sperm viability in the ejaculate was performed by diluting 1 in 500 with the polymer-free medium. The post-CCCD recovered samples were assayed without further dilution. 


\section{CCCD analysis}

Preparation of cell samples. After standard sperm evaluation, seminal plasma was removed to avoid its influence in the CCCD assay, by filtering twice through a $5 \mu \mathrm{m}$ pore size Millipore disk (Millipore Corp., Bedford, $\mathrm{MA}$ ) and mixing the sample with 20 volumes of the CCCD polymer-free medium.

Two-phase system. The two-phase system used consisted of 5.5\% (w/w) dextran T500, 2\% (w/w) PEG 6000, 10.5\%

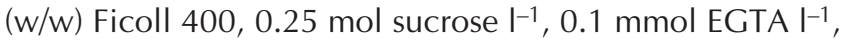
4 mmol sodium phosphate $\mathrm{I}^{-1}, \mathrm{pH} 7.5,5 \mathrm{mmol}$ glucose $\mathrm{I}^{-1}$, $10 \mathrm{mmol}^{-}$Hepes $\mathrm{I}^{-1}$ and $2 \mathrm{mmol} \mathrm{KOH} \mathrm{I}^{-1}$, final $\mathrm{pH} 7.5$.

Centrifugal countercurrent distribution. The countercurrent distribution machine was designed based on the one described by Akerlund (1984). The apparatus consists of a rotor containing two concentric rings in which 60 chambers are arranged in a circle. Half of each chamber is in the outer ring and the other half is in the inner ring, and the two rings can rotate against each other. Each chamber contains a mix of the two phases. Centrifugation is used to separate the phases so that the denser (bottom) phase is in the outer half of each chamber and the lighter (upper) phase is in the inner half. During the centrifugation stage, the inner ring rotates against the outer ring so that the upper phase of one chamber is transferred to the next chamber. The overall process consists of a multi-step transfer of 60 upper over 60 bottom batch phases. A detailed scheme of the device has been reported by Akerlund (1984).

A two-phase system of the above composition was prepared and mixed to perform the CCCD experiments. Batches of $400 \mathrm{~g}$ were assembled by weighing out stock solutions to avoid differences among experiments. Thus, three experiments were performed using the same system, in identical conditions, allowing direct comparison among six different samples. Two cell samples (each approximately $1.2 \times 10^{8}$ cells) obtained after the filtration process were loaded simultaneously in chambers 0 and 30, and analysed in identical conditions by performing 29 transfers (the whole process takes approximately $1 \mathrm{~h}$ ). All experimentation was performed at $20^{\circ} \mathrm{C}$. After the run, the content of each chamber was diluted with an equal volume of polymer-free medium to yield a single-phase suspension, which was collected and the cells were counted under a light microscope.

Partition results were expressed as a percentage of the cells counted in each fraction with respect to the value obtained in the chamber containing the highest number of cells. For viability assessment, cells from three consecutive chambers throughout the run were pooled and stained as indicated above. Results were expressed as the percentage of viable cells in each sample. As a consequence of the separation procedure, populations of sperm cells with a marked affinity for the lower dextran-rich phase (mainly a result of low hydrophobicity) partition in the left part of the profile. Sperm cells that partition almost equally in both phases distribute in the central sector. Lastly, sperm populations with a high affinity for the upper PEG-rich phase (mainly a result of high hydrophobicity), partition in the right-hand sector of the profile.

\section{Experimental design and presentation of data}

A total of 522 ewes from 26 different farms was inseminated with 53 ejaculates obtained from 25 rams. Fertility was assessed as the percentage of deliveries with respect to the total number of inseminated ewes. One sample of each ejaculate was used for standard semen and CCCD analysis. The routine parameters evaluated were sperm motility, sperm concentration and cell viability, before and after filtration. Post-CCCD parameters were derived from the analysis of the obtained profile after CCCD: the total number of viable cells recovered after the CCCD process (TV) was represented as a dark area which corresponds to the $\Sigma_{0}^{29} \%$ viable cells in each chamber with respect to the number of cells in the chamber containing the maximum (in the largest sperm subpopulation). The $T V$ was also divided into three fractions $\left(V_{1}, V_{2}\right.$ and $V_{3}$, corresponding to chambers 0-9, 10-19 and 20-29, respectively). This partition was made according to our previous results, which have shown that non-viable cells are located preferentially in the left-hand side of the profile, where acrosome-reacted and immature spermatozoa also partition; however, viable spermatozoa are mainly partitioned in the central and right-hand sectors of the profile. The heterogeneity of the profile $(H)$ was defined as the percentage of chambers containing a number of cells $\geqslant 50 \%$ of that in the chamber containing the maximum number.

\section{Statistical analysis}

Statistical analyses were undertaken using SPSS software (version 6.1.2; 1995). Correlations between sperm parameters and field fertility were calculated using Pearson's coefficient.

Stepwise multiple regression analysis was used for determining the variation in field fertility to estimate regression equations to predict the fertility percentage on the basis of measures of sperm parameters. ANOVAs were performed to determine whether there were significant differences between groups in some of the above mentioned parameters.

\section{Results}

\section{Relationship between standard semen analyses and} in vivo fertility

No significant correlation was found between the standard parameters analysed and field fertility achieved with the 53 ejaculates studied (Table 1). 

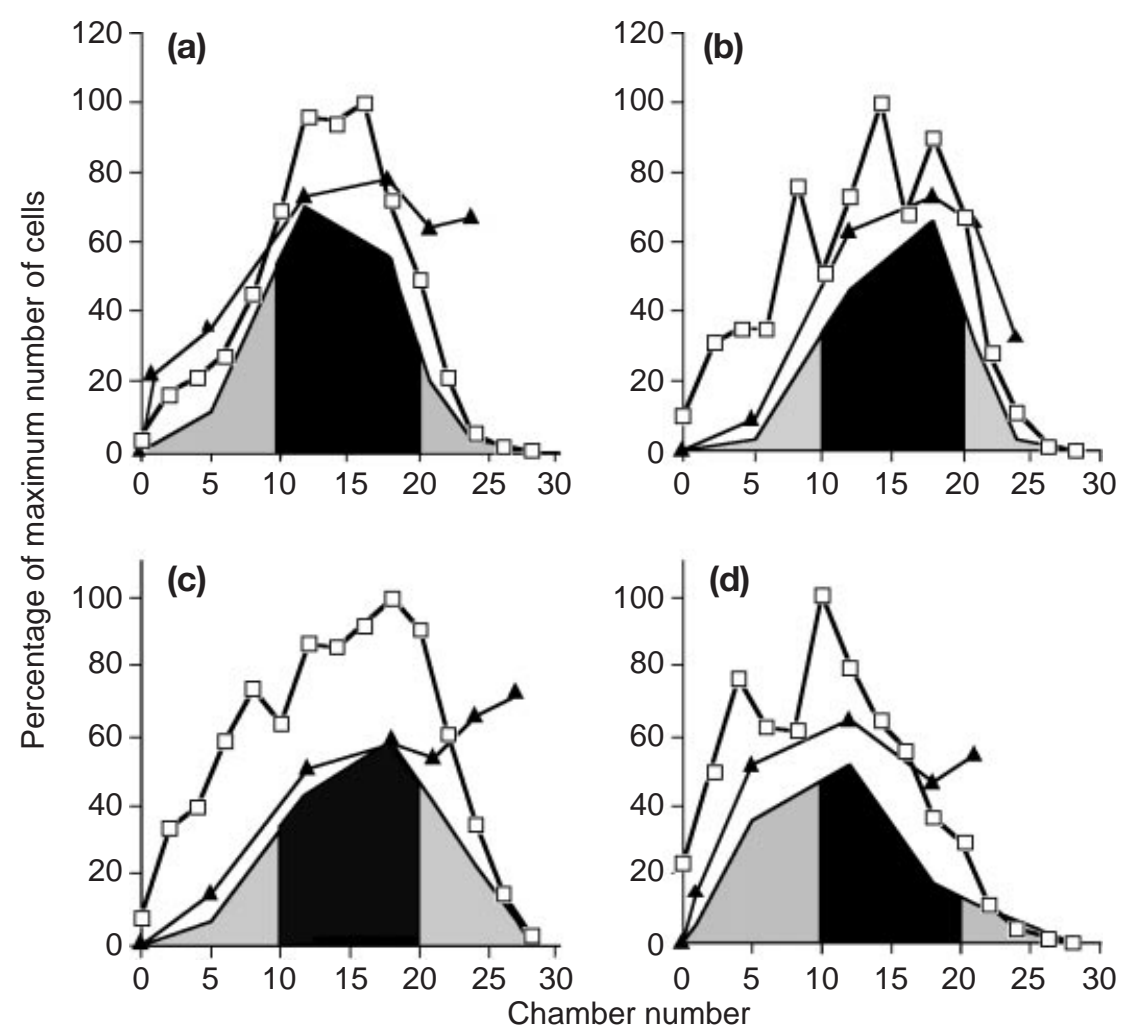

Fig. 1. Representative centrifugal countercurrent distribution analysis (CCCD) profiles of high fertility ram ejaculates, corresponding to (a) $75.00 \%$, (b) $69.20 \%$, (c) $62.00 \%$ and (d) $57.14 \%$ of field fertility. $\square$ : Percentage of maximum number of cells; $\boldsymbol{\Delta}$ : percentage of viable cells; solid black area is total viable cells in central area $\left(V_{2}\right)$; grey-shaded area is total viable cells in $V_{1}$ and $V_{3}$; total black + grey-shaded area is total viable cells in all chambers.

\section{Relationship between post-CCCD analysis parameters and in vivo fertility}

The parameters derived from the analysis of the CCCD profiles were: the recovered viability, both total (TV) and

Table 1. Correlations between single semen parameters and ram fertility in vivo

\begin{tabular}{lcc}
\hline Parameters & $r$ & $P$ value \\
\hline Standard semen analyses & & \\
$\quad$ Concentration & -0.0114 & $\mathrm{~ns}$ \\
Mass motility & -0.0225 & $\mathrm{~ns}$ \\
Individual motility & -0.0341 & $\mathrm{~ns}$ \\
Initial viability & -0.0722 & $\mathrm{~ns}$ \\
Post-filtration viability & 0.1348 & $\mathrm{~ns}$ \\
& & \\
Post-CCCD analyses & & \\
Total viability $(T V)$ & 0.4006 & $<0.005$ \\
Viability chambers $0-9\left(V_{1}\right)$ & 0.2365 & $<0.1$ \\
Viability chambers 10-19 $\left(V_{2}\right)$ & 0.4152 & $<0.005$ \\
Viability chambers 20-29 $\left(V_{3}\right)$ & 0.0102 & $\mathrm{~ns}$ \\
Heterogeneity & 0.2116 & $<0.1$ \\
\hline
\end{tabular}

ns: not significant. divided into three fractions $\left(V_{1}, V_{2}\right.$ and $\left.V_{3}\right)$, and the heterogeneity of the profile $(H)$. The correlation coefficients between these parameters and field fertility, considering all the data obtained from the 53 ejaculates, are shown (Table 1). The recovered viability showed the highest correlation with fertility, particularly in the central chambers $\left(V_{2}\right.$, $r=0.415, P<0.005)$. The $V_{2}$ value ranged from 193.3 to 591.1 among all ejaculates. Heterogeneity of the ejaculate revealed by CCCD also showed a positive correlation with field fertility $(r=0.23)$, with a tendency towards significance $(P<0.1)$. The variability of this parameter among all ejaculates ranged from $20.00 \%$ to $86.66 \%$.

\section{Representative CCCD profiles of ejaculates with different field fertility rates}

The mean fertility of all ejaculates used in this study was $46.75 \%$, ranging from $12.5 \%$ to $75.0 \%$. As a result of this large difference in fertility, ejaculates were classified in two categories according to the fertility obtained: higher ( $>46.75 \%$ ) and lower $(<46.75 \%)$ than the mean fertility value.

Four CCCD representative profiles of high fertility ejaculates are shown (Fig. 1). These profiles showed, as a common characteristic, a very high viability value, especially in the central chambers $\left(V_{2}\right)$, ranging from 591.1 


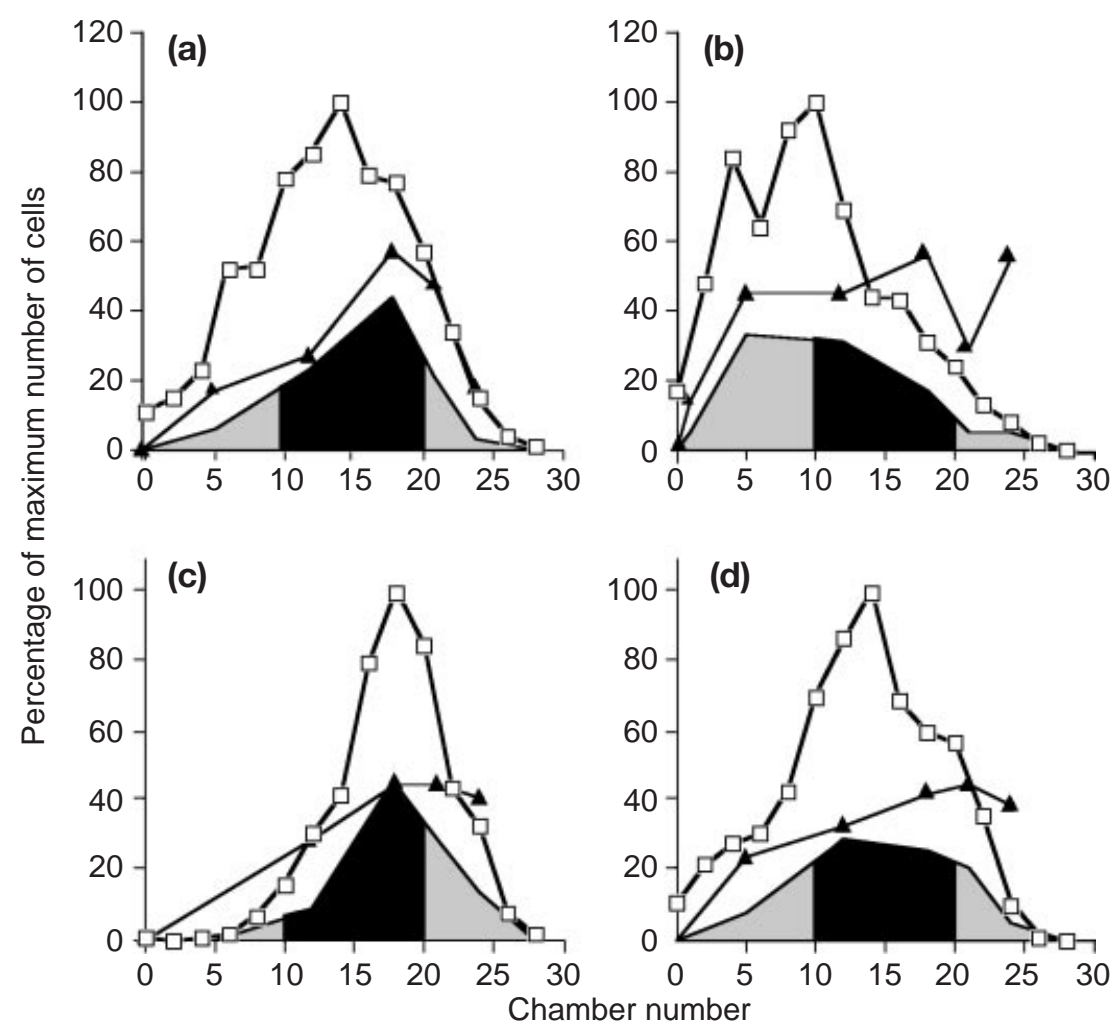

Fig. 2. Representative centrifugal countercurrent distribution analysis (CCCD) profiles of low fertility ram ejaculates, corresponding to (a) $41.66 \%$, (b) $28.57 \%$, (c) $16.66 \%$ and (d) $14.28 \%$ of field fertility. $\square$ : Percentage of maximum number of cells; $\boldsymbol{\Delta}$ : percentage of viable cells; solid black area is total viable cells in central area $\left(V_{2}\right)$; grey-shaded area is total viable cells in $V_{1}$ and $V_{3}$; total black + grey-shaded area is total viable cells in all chambers.

to 478.6 , with the exception of the sample in Fig. $1 \mathrm{~d}$. This ejaculate achieved a $57.14 \%$ field fertility despite its lower $V_{2}$ value $(417.2$, Table 2a). This could be explained by its relatively high heterogeneity $(46.66 \%$, Table $2 \mathrm{a})$, which could compensate for its lower viability.

Four CCCD representative profiles of low fertility ejaculates are also shown (Fig. 2). $V_{2}$ ranged from 298.1 to

Table 2. Field fertility, $V_{2}$ and heterogeneity values corresponding to centrifugal countercurrent distribution analysis (CCCD) profiles for the ram ejaculates shown in Fig. 1 and Fig. 2

\begin{tabular}{|c|c|c|c|}
\hline & fertility $(\%)$ & $V_{2}$ & Heterogeneity (\%) \\
\hline \multirow[t]{4}{*}{ (a) High fertility (Fig.1) } & 75.00 & 591.11 & 33.33 \\
\hline & 69.23 & 505.88 & 46.66 \\
\hline & 62.00 & 478.67 & 60.00 \\
\hline & 57.14 & 417.26 & 46.66 \\
\hline \multirow[t]{4}{*}{ (b) Low fertility (Fig. 2) } & 41.66 & 298.10 & 46.66 \\
\hline & 28.57 & 246.65 & 33.33 \\
\hline & 16.66 & 241.32 & 20.00 \\
\hline & 14.28 & 241.52 & 40.00 \\
\hline
\end{tabular}

$V_{2}$ is the recovered viability in the CCCD profile central chambers
241.3 (Table 2b) and was, in all cases, lower than the $V_{2}$ of high fertility samples (Fig. 1). Likewise, heterogeneity was also lower than in ejaculates of higher fertility (Table 2b).

ANOVA were performed to determine whether there were significant differences between both groups in some of the above mentioned parameters. Only the viability recovered in the central chambers $\left(V_{2}\right)$ was a parameter with a predictive capacity to discriminate between the two groups $(P<0.05)$.

\section{Relationship between predictive fertility and observed field fertility}

Taking into account both parameters at once from all ejaculates analysed, a significant correlation with field fertility was found, leading to a predictive equation for field fertility with a correlation coefficient $r=0.488$ and a high level of significance $(P<0.005)$ :

$$
P F=6.02+0.069 V_{2}+0.315 H
$$

The significance of the parameters, the recovered viability in the CCCD profile central chambers (V2) and heterogeneity $(\mathrm{H})$, in the equation was $P<0.005$ and $P<0.05$, respectively. 


\section{Discussion}

In the present study, no correlation was found between cell viability of the ejaculate and fertility. However, the recovered cell viability after CCCD, expressed both as total ( $T V$ ) and in the central chambers $\left(V_{2}\right)$, is the parameter that showed the highest correlation with fertility. This could be a result of the effects of the CCCD process, which could cause some damage to the cells, reflected in the viability loss of those cells more liable to suffer membrane alterations. Thus, recovered viability, especially $V_{2}$, could reflect the resistance of the spermatozoa to stressful events, including those that would occur during insemination and the passage through the female genital tract. As suggested by Shannon (1978), differences in the ability of spermatozoa to survive in the female tract during the interval between insemination and ovulation could produce differences in fertility. This could be the explanation for the lack of correlation between initial viability and fertility, as it would not be able to reflect this resistance, in contrast to viability recovered after CCCD.

Furthermore, the viability of the central chambers $\left(V_{2}\right)$ allows discrimination between ejaculates with field fertility higher or lower than the mean fertility, as shown by ANOVA. Therefore, the practical advantage of the CCCD technique is considerable as it provides a fast method for discarding ejaculates with low fertilizing capacity.

Spermatozoa in an ejaculate are very heterogeneous in their attributes, including fertilizing capacity. This heterogeneity is very important in species in which there is a variable and unpredictable interval between mating, or artificial insemination, and ovulation (Amann and Hammerstedt, 1993), as in sheep. Heterogeneity is acquired during sperm maturation and provides different populations of spermatozoa, which, in turn, differ in their fertilizing capability. Hence, the heterogeneity evaluation of an ejaculate could provide important information about the fertilizing potential of a given sample.

Ollero et al. (1994) showed the ability of CCCD to assess the intrinsic heterogeneity of a ram ejaculate.

As many of the steps that occur during fertilization depend on changes in the surface of spermatozoa, CCCD could be very useful in predicting the fertilizing potential of an ejaculate, on the basis of its heterogeneity, as well as on other parameters derived from the CCCD profile.

In addition to the importance of the heterogeneity of a semen sample, the significance of membrane integrity cannot be ruled out. Although a single ejaculate presents a high number of cell populations differing in their maturation state, if the viability of these populations is very low, it is likely that the fertilizing ability of the ejaculate will be poor. Furthermore, the heterogeneity of the sample is not capable, on its own, of discriminating between ejaculates of fertility higher or lower than the mean, as $V_{2}$ is. This fact indicates that, despite the relevance of the heterogeneity of an ejaculate, this parameter has to be considered together with $V_{2}$. Thus, a regression equation including both parameters could be used to predict field fertility of an ejaculate. Although the predictive equation does not show a very high correlation coefficient, it has a very high level of significance. This could be a result of the number of external factors affecting results, such as females from different farms, distance from the sire station to the insemination points and technical insemination equipment. When a similar study was undertaken by our team with control ewes, located at the same farm, and inseminated by the same technical equipment via the intrauterine route, the correlation between these two parameters and fertility was very much higher (MuiñoBlanco et al., 2000).

The lack of correlation between standard semen analysis and field fertility must also be considered. Initial concentration should not be taken into consideration as the number of spermatozoa per straw is adjusted to a fixed value, with the same number of spermatozoa inseminated in all cases. Motility, both mass or individual, estimated subjectively, has also shown no correlation with fertility in several studies (Eriksson et al., 1994; Januskauskas and Rodriguez-Martinez, 1995), in contrast with computerassisted sperm analysis (CASA), which showed significant correlation (Correa et al., 1997; Zhang et al., 1998). The lack of correlation between initial viability and fertility is in agreement with results from several reports (Eriksson et al., 1994; Januskauskas et al., 1995; Garner et al., 1997). Although correlation between non-return rate and viability, expressed as the number of viable spermatozoa multiplied by the total number of spermatozoa, has been reported (Pace et al., 1981), other authors have indicated that it may be a result of the total dose only, as the multiplication is necessary to detect the correlation (Fearon and Wegener, 2000).

In conclusion, CCCD is a technique that could be considered as a useful tool in estimation of the fertilizing potential of a given semen sample. Viability values in the central chambers of the CCCD profile, taken together with the heterogeneity of the ejaculate, provide a method for the allocation of measures of semen quality to an appropriate level. Moreover, it might be used in large breeding centres for discarding ejaculates with low fertilizing capacity, as well as for determining the value of particular males in artificial insemination programmes. A number of ejaculates representative of that sire, between 6-10 ejaculates, obtained with a regular extraction system of two ejaculates per week over 1 month, would be suitable for a reliable analysis.

This work was supported by grants DGA-P 48/99 AV and CICYT AGL-00-1221. R. Pérez-Pé had a fellowship from the DGA. The authors thank E. Vigil (CENSYRA, Zaragoza) for supplying the semen samples and P. Quintín for his technical assistance.

\section{References}

Akerlund HE (1984) An apparatus for counter-current distribution in a centrifugal acceleration field Journal of Biochemical and Biophysical Methods 9 133-141 
Albertsson PA (1986) Partition of Cell Particles and Macromolecules John Wiley and Sons, New York

Amann RP and Hammerstedt RH (1993) In vitro evaluation of sperm quality: an opinion Journal of Andrology 14 397-406

Barth AD (1992) The relationship between sperm abnormalities and fertility Proceedings of the 14th Technical Conference on Artificial Insemination and Reproduction 47-63

Correa JR, Pace MM and Zavos PM (1997) Relationships among frozen-thawed sperm characteristics assessed via the routine semen analysis, sperm functional tests and fertility of bulls in an artificial insemination program Theriogenology 48 721-731

Eriksson B, Shamsuddin M, Haard M, Larsson B, Söderquist L and Rodriguez-Martinez H (1994) Motility and membrane integrity of frozen-thawed bull spermatozoa in relation to IVF and field fertility Proceedings of the XVII Nordic Veterinary Congress 2 200-201

Fazeli AR, Holt C, Steenweg W, Bevers MM, Holt WV and Colenbrander B (1995) Development of a sperm hemizona binding assay for boar semen Theriogenology 44 17-27

Fazeli AR, Zhang BR, Steenweg W, Larsson B, Bevers MM, van der Broek J, Rodriguez-Martinez H and Colenbrander B (1997) Relationship between sperm-zona pellucida binding assays and the 56-day nonreturn rate of cattle inseminated with frozen-thawed bull semen Theriogenology 48 853-863

Fearon JM and Wegener PT (2000) Relationship between fertility in cattle and the number of inseminated spermatozoa Journal of Reproduction and Fertility 119 293-308

Fisher D and Sutherland IA (1989) Separation Using Aqueous Phase Systems. Applications in Cell Biology and Biotechnology Plenum Press, New York and London

Gadea J, Matás C and Lucas X (1998) Prediction of porcine semen fertility by homologous in vitro penetration (hIVP) assay Animal Reproduction Science 56 95-108

García-López N, Ollero M, Cebrián-Pérez JA and Muiño-Blanco T (1996) Reversion of thermic-shock effect on ram spermatozoa by adsorption of seminal plasma proteins revealed by partition in aqueous two-phase systems Journal of Chromatography B $\mathbf{6 8 0} 137-143$

Garner DL, Thomas CA, Allen CH, Senger PL and Sasser RG (1997) Effect of cryopreservation on bovine sperm viability as determined by dual DNA staining Reproduction in Domestic Animals 32 279-283

Graham EF, Schmehl MKL, Evenson BK and Nelson DS (1980) Problems with laboratory assays Proceedings of the 8th Technical Conference on Artificial Insemination and Reproduction, National Association of Animal Breeders, Wisconsin 1-8

Harrison RAP and Vickers SE (1990) Use of fluorescent probes to assess membrane integrity in mammalian spermatozoa Journal of Reproduction and Fertility 88 343-352

Harrison RAP, Dott HM and Foster GC (1982) Bovine serum albumin, sperm motility, and the "dilution effect" Journal of Experimental Zoology 222 81-88

Holt C, Holt WV, Moore HDM, Reed HCB and Curnock RM (1997) Objectively measured boar sperm motility parameters correlate with the outcomes of on-farm inseminations: results of two fertility trials Journal of Andrology $18312-323$

Ivanova $\mathbf{M}$ and Mollova M (1993) Zona-penetration in vitro test for evaluating boar sperm fertility Theriogenology 40 397-410

Januskauskas A and Rodriguez-Martinez H (1995) Assessment of sperm viability by measurement of ATP, membrane integrity and motility in frozen/thawed bull semen Acta Veterinaria Scandinavica 36 571-574

Januskauskas A, Söderquist L, Haard MC, Lundeheim N and RodriguezMartinez H (1996) Influence of sperm number per straw on the post- thaw sperm viability and fertility of Swedish Red and White Al bulls Acta Veterinaria Scandinavica 37 461-470

Januskauskas A, Gil J, Söderquist L, Haard MGM, Haard MC, Johannisson A and Rodriguez-Martinez H (1999) Effect of cooling rates on post-thaw sperm motility, membrane integrity, capacitation status and fertility of dairy bull semen used for artificial insemination in Sweden Theriogenology 52 641-658

Kjaestad H, Ropstad E and Berg KA (1993) Evaluation of spermatological parameters used to predict the fertility of frozen bull semen Acta Veterinaria Scandinavica 34 299-303

Meyers SA, Liu IKM, Overstreet JW, Vadas S and Drobnis EZ (1996) Zona pellucida binding and zona-induced acrosome reactions in horse spermatozoa: comparisons between fertile and subfertile stallions Theriogenology 46 1277-1288

Muiño-Blanco T, Pérez-Pé R, Báguena O, Grasa P, Abecía JA, Forcada F and Cebrián-Pérez JA (2000) Sperm selection by a dextran/swim up procedure increases the embryo rates following in vitro fertilization by intrauterine insemination in superovulated ewes. Prediction of fertility by centrifugal countercurrent distribution (CCCD) in an aqueous two-phase system 25th Annual Meeting of the American Society of Andrology 67 (Abstract)

Ollero M, Pascual ML, Muiño-Blanco T, Cebrián-Pérez JA and López-Pérez MJ (1994) Revealing surface changes associated with maturation of ram spermatozoa by centrifugal counter-current distribution in an aqueous two-phase system Journal of Chromatography A 668 173-178

Ollero M, Cebrián-Pérez JA and Muiño-Blanco T (1997) Improvement of cryopreserved ram sperm heterogeneity and viability by addition of seminal plasma Journal of Andrology 18 732-739

Pace MM, Sullivan JJ, Elliott FI, Graham EF and Coulter GH (1981) Effects of thawing temperature, number of spermatozoa and spermatozoal quality on fertility of bovine spermatozoa packaged in $0.5 \mathrm{ml}$ French straws Journal of Animal Science 53 693-701

Pérez LJ, Valcárcel A, de las Heras MA and Baldassarre H (1997) Comparative study of four techniques for evaluation of sperm quality in ovine and bovine frozen-thawed samples Reproduction in Domestic Animals 32 157-160

Rogers B, Bentwood B, Campen H, Helmbrecht G, Soderdahl D and Hale R (1983) Sperm morphology assessment as an indicator of human fertilizing capacity Journal of Andrology 4 119-125

Shannon P (1978) Factors affecting semen preservation and conception rates in cattle Journal of Reproduction and Fertility 54 519-527

Söderquist L (1991) Sperm Characteristics and Fertility in Dairy Al Bulls PhD Thesis, University of Uppsala

Tatemoto H, Horiuchi T, Maeda T, Terada T and Tsutsumi Y (1994) Penetration by bull spermatozoa into the zona pellucida of dead bovine oocytes recovered from frozen-thawed ovaries Theriogenology 42 $465-474$

Whitfield CH and Parkinson TJ (1995) Assessment of the fertilizing potential of frozen bovine spermatozoa by in vitro induction of acrosome reactions with calcium ionophore (A23187) Theriogenology 44 413-422

Zhang BR, Larsson B, Lundeheim N and Rodriguez-Martinez H (1998) Sperm characteristics and zona pellucida binding in relation to field fertility of frozen-thawed semen from dairy AI bulls International Journal of Andrology 21 207-216

Received 16 May 2001.

First decision 27 July 2001.

Revised manuscript received 28 December 2001.

Accepted 10 January 2002. 